Review

\title{
Structure and function of regulatory RNA elements: Ribozymes that regulate gene expression
}

\author{
William G. Scott ${ }^{\mathrm{a}, *}$, Monika Martick ${ }^{\mathrm{a}}$, Young-In Chi ${ }^{\mathrm{b}}$ \\ a Center for the Molecular Biology of RNA, Sinsheimer Laboratory, University of California at Santa Cruz, Santa Cruz, CA, USA \\ ${ }^{b}$ Center for Structural Biology, Department of Molecular and Cellular Biochemistry, University of Kentucky, Lexington, KY, USA
}

\section{A R T I C L E I N F O}

Article history:

Received 19 May 2009

Received in revised form 1 September 2009

Accepted 13 September 2009

Available online 23 September 2009

\section{Keywords:}

Ribozyme

Riboswitch

RNA gene regulation

\begin{abstract}
A B S T R A C T
Since their discovery in the 1980s, it has gradually become apparent that there are several functional classes of naturally occurring ribozymes. These include ribozymes that mediate RNA splicing (the Group I and Group II introns, and possibly the RNA components of the spliceosome), RNA processing ribozymes (RNase P, which cleaves precursor tRNAs and other structural RNA precursors), the peptidyl transferase center of the ribosome, and small, self-cleaving genomic ribozymes (including the hammerhead, hairpin, HDV and VS ribozymes). The most recently discovered functional class of ribozymes include those that are embedded in the untranslated regions of mature mRNAs that regulate the gene's translational expression. These include the prokaryotic glmS ribozyme, a bacterial riboswitch, and a variant of the hammerhead ribozyme, which has been found embedded in mammalian mRNAs. With the discovery of a mammalian riboswitch ribozyme, the question of how an embedded hammerhead ribozyme's switching mechanism works becomes a compelling question. Recent structural results suggest several possibilities.
\end{abstract}

(c) 2009 Elsevier B.V. All rights reserved.

\section{Background and context}

\subsection{Discovery of ribozymes}

RNA catalytic activity in the absence of proteins was first observed in the context of Group I intron self-splicing [1], and the first example of a true RNA enzyme (or ribozyme) that exhibits multiple turnover was the RNA subunit of RNase P, which hydrolyzes the 5'-end of precursor tRNAs [2]. Subsequently, a number of smaller and chemically similar self-cleaving genomic RNAs were discovered [3-5]. The structures of the prokaryotic ribosome [6-11] confirmed an earlier suggestion that the peptidyl transferase center was comprised entirely of RNA [12]; no ribosomal protein approaches closer than 18 Å to the active site. Subsequent to the discovery of bacterial riboswitches [13], a prokaryotic ribozyme riboswitch has been identified in the form of a new ribozyme (glmS) [14], and a familiar ribozyme (the genomic hammerhead ribozyme that mediates rolling-circle replication in RNA virus-like genomes) has been identified, as a discontinuous sequence, embedded in mammalian mRNAs [15], a realm wherein riboswitches have not yet been identified.

\subsection{Chemistry of ribozyme catalysis}

With the exception of the peptidyl transferase, all of the naturally occurring ribozymes employ simple variations of acid/base catalyzed

\footnotetext{
* Corresponding author.

E-mail address: wgscott@chemistry.ucsc.edu (W.G. Scott).
}

phosphodiester chemistry, and all but the hydrolytic RNase $\mathrm{P}$ catalyze a phosphodiester isomerization reaction that breaks or joins the RNA backbone [16]. Each ribozyme, however, has a completely unique sequence, tertiary structure, and detailed catalytic strategy, sharing in common only the general principles of acid/base catalysis [17]. Several of the small, self-cleaving genomic RNAs catalyze a chemically identical site-specific self-cleavage reaction in which the 2'-oxygen attacks an adjacent phosphate, producing products with $5^{\prime}-\mathrm{OH}$ and $2^{\prime}, 3^{\prime}$-cyclic phosphate termini; the reaction is the same as the first step catalyzed by RNase A (Fig. 1). As with RNase A, most of these ribozymes (the exception is the HDV ribozyme) do not require the presence of a divalent metal ion for catalysis [18]. The glmS riboswitch ribozyme also catalyzes the same self-cleavage reaction.

\subsection{Comparison to a protein enzyme: RNase A}

RNase A provides a convenient and familiar reference point with respect to protein mechanistic enzymology for understanding ribozyme acid/base catalysis $[19,20]$. Two invariant histidines in the active site of RNase A function as a general base and a general acid. In the first step of the RNase A cleavage reaction, an unprotonated histidine (His 12) abstracts a proton from the $2^{\prime}-\mathrm{OH}$ of the substrate RNA at the cleavage site, thus generating the nucleophile that attacks the adjacent phosphate, and a protonated histidine (His 119) donates a proton to the $5^{\prime}$-oxygen leaving group, which begins to accumulate an unstable negative charge as its bond to the scissile phosphorus atom breaks. A bond between the 2'-oxygen, the attacking 
nucleophile, and the phosphorus forms at the same time the scissile bond breaks, as the reaction passes through a pentacoordinated oxyphosphorane transition state in which the attacking nucleophile and leaving group oxygen occupy the two opposite, axial positions. The products are two strands of RNA; one with a $5^{\prime}-\mathrm{OH}$ terminus and the other with a $2^{\prime}, 3^{\prime}$-cyclic phosphodiester (The second step of the RNase A reaction involves hydrolysis of the cyclic phosphate).

Each of the small self-cleaving ribozymes employs a unique catalytic strategy to perform acid/base catalysis, but the chemical mechanism of the cleavage reaction is the same as the fist step of the RNase A reaction, and the pentacoordinated transition-state structure appears to be essentially the same. Obviously, ribozymes lack histidines in their active sites, but various RNA functional groups appear to play analogous roles as general acids and bases [21].

In the case of the hairpin ribozyme, a deprotonated guanosine appears to be the general base that plays the analogous role to the deprotonated histidine in RNase A, and a (protonated) adenosine appears to be the general acid [22]. In the case of the hammerhead ribozyme, a deprotonated guanosine also appears to be the general base, but instead of adenosine, the $2^{\prime}-\mathrm{OH}$ of a conserved nucleotide appears to function as a general acid (Fig. 1) [23]. In the case of the glmS ribozyme, guanosine again appears to be the base, whereas the acid catalyst is not the RNA itself, but rather the sugar, GlcN6P, the small molecule riboswitch effector, that binds specifically to the active site of the ribozyme and activates it $[24,25]$.

\subsection{The biological context of genomic RNA catalysis}

The hammerhead ribozyme was the first of several catalytic RNAs to be discovered in the context of satellite RNA virus and virus-like genomes [3]. With the exception of the human hepatitis $C$ virus, these were all discovered in plants [26]. The hammerhead ribozyme was first found in the sense strand of the satellite RNA of tobacco ringspot virus, which was known to replicate via a rolling-circle pathway. Arguably the simplest nucleic acid replicative strategy, the singlestranded, covalently closed circular satellite RNA serves as a substrate for a host RNA polymerase. As the polymerase circumnavigates the satellite RNA template for multiple processive cycles, it produces a linear concatomeric complementary copy of the circular RNA. The concatomer must be cleaved into monomeric fragments and then the ends of the monomers must ligate to form circular templates for the second half of replication [27].

The hammerhead ribozyme catalyzes the cleavage (and possibly ligation) reactions for the sense-strand RNA, and the subsequently identified hairpin ribozyme catalyzes cleavage and ligation of the complementary template. In addition to plant RNA viruses, active hammerhead sequences are occasionally found in satellite RNA transcripts of highly repetitive DNA sequences in organisms such as Schistosoma; the function of these satellite RNAs is unknown $[28,29]$.

Because rolling-circle replication of genomic RNAs requires both an RNA cleavage and an RNA ligation event, it is quite understandable why a phosphodiester isomerization reaction might be preferred, in natural selection, over an RNA hydrolysis reaction. Formation of the $2^{\prime}, 3^{\prime}$-cyclic phosphate preserves the initial state of the scissile phosphate as a phosphodiester, and thus permits it to function as a substrate in a subsequent ligation reaction without the requirement for a high-energy cofactor such as ATP. Simple RNA hydrolysis would be unable to do that. It is therefore not particularly surprising that all of the various genomic ribozymes (including the hairpin, hammerhead, HDV and VS ribozymes) catalyze the same chemical reaction. The mechanisms of the switch between nuclease and ligase activities for the genomic ribozymes remains completely elusive, although structural analyses of the hammerhead ribozyme, described below, are beginning to offer some insights.

\section{The hammerhead ribozyme}

\subsection{The structural basis for hammerhead ribozyme catalysis}

Three-dimensional structures now exist for the hairpin, HDV and hammerhead ribozymes, each in both pre-cleavage and post-cleavage states [21,22,30-33]. Each of these ribozymes possesses a unique sequence and tertiary structure as well as a unique catalytic strategy for accelerating the same reaction; the one common theme is that all appear to use a form of acid/base catalysis as their primary mode of rate enhancement. For purposes of continuity, we will focus here on the hammerhead ribozyme.

Hammerhead RNA self-cleavage motifs consist of three A-form helices (called Stems I, II and III) flanking a junction comprised of 15 invariant or mostly conserved nucleotides that form the catalytic core (Fig. 2a) [34]. Two of the three helices (typically Stem II and Stem I) are capped by connecting loops, giving this self-cleaving RNA sequence a canonical secondary structure that resembles the shape of a hammer head. The natural full-length hammerhead ribozyme sequence typically contains an additional tertiary contact between Stems I and II, distant from the cleavage site (Fig. 2b), that greatly enhances the rate of catalysis by stabilizing formation of the active conformation of the cleavage site (Fig. $2 c$ and d) [23,35,36].

The crystal structure of the full-length hammerhead ribozyme sequence derived from Schistosoma mansoni $(\operatorname{Sm} \alpha)$ was obtained in 2006 (2GOZ) [23]. Unlike previous structures of minimal hammerhead ribozyme sequences that lacked the distal tertiary contact, this structure reveals the active conformation of the cleavage site.

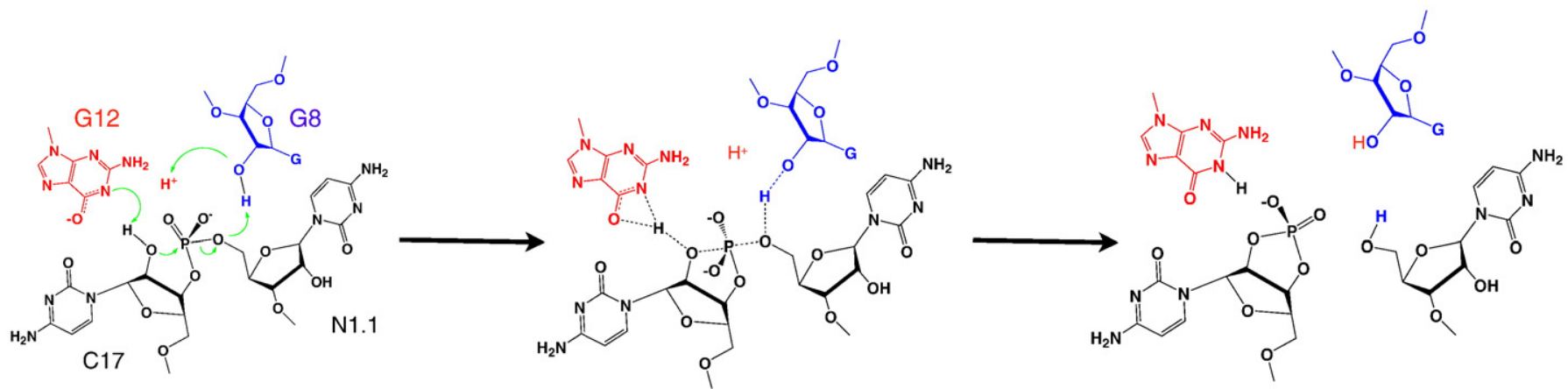

Enzyme-Substrate Complex

Transition-State

Enzyme-Product Complex

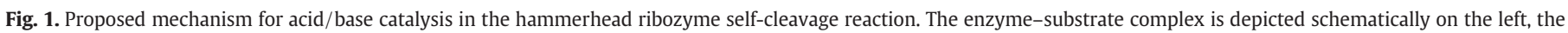
putative transition-state structure in the center, and the enzyme-product complex is on the right. 
a

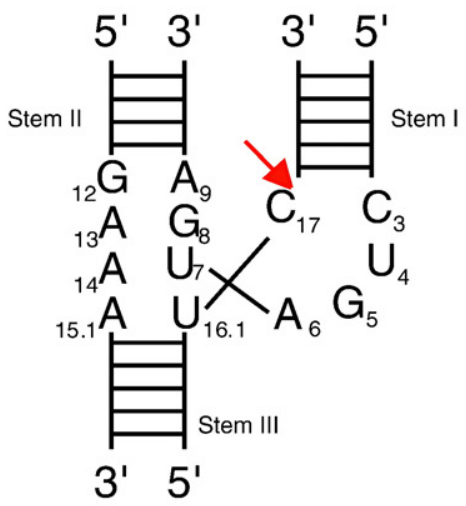

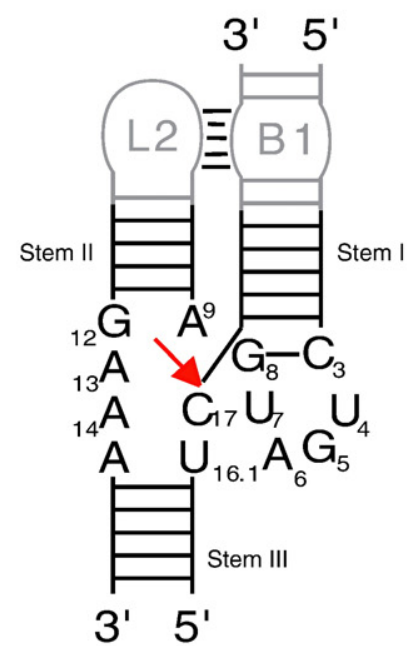

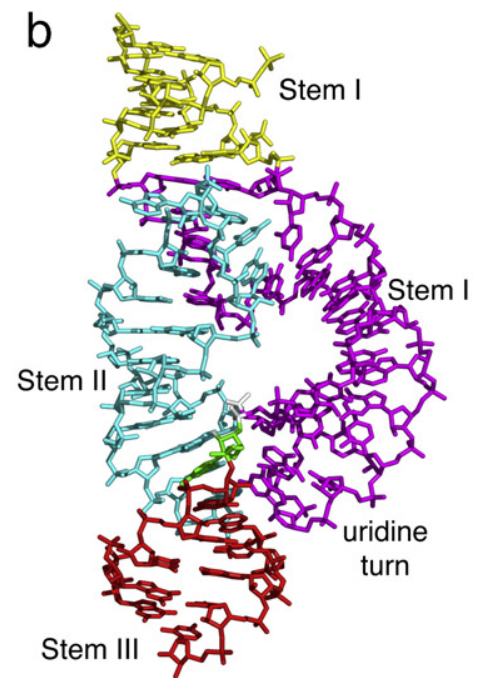

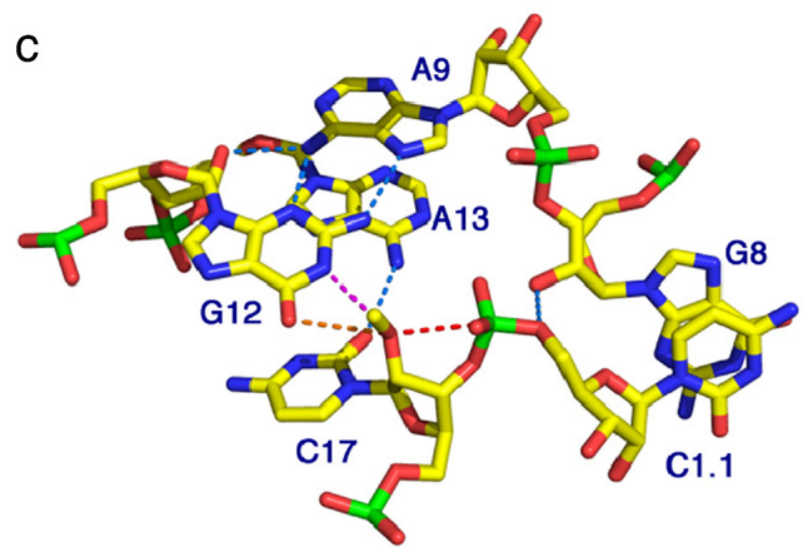

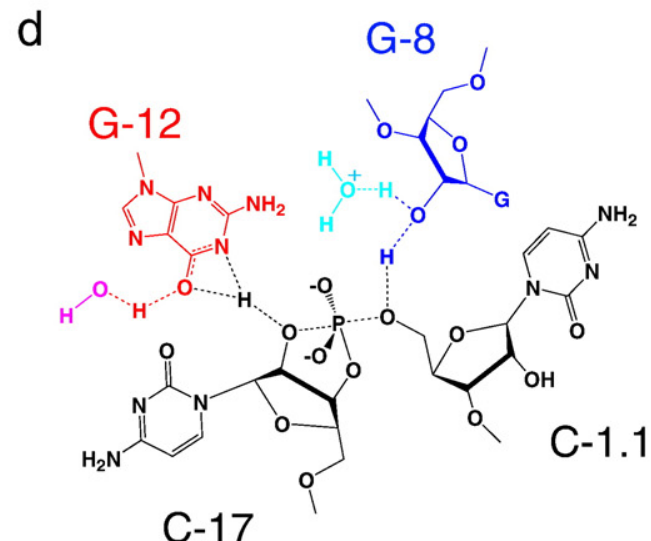

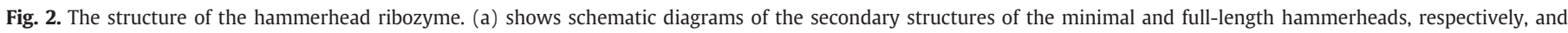

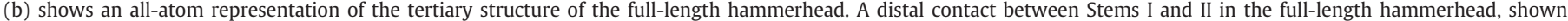
schematically in (a), stabilizes the active site structure, the details of which are shown in (c). (d) shows a proposed transition state structure extrapolated from (c).

Specifically, the invariant nucleotide $\mathrm{G} 12$ is positioned with respect to the cleavage site in a manner consistent with its proposed role in general base catalysis in the hammerhead cleavage reaction, and the $2^{\prime}-\mathrm{OH}$ of the invariant G8 is positioned in a manner consistent with a role in acid catalysis, i.e., protonation of the $5^{\prime}$-oxygen leaving group. The cleavage-site nucleotide and the adjacent phosphate are both positioned in a manner consistent with the known in-line attack chemical mechanism, and many other biochemically inferred characteristics are readily explained from the structure. The details of the active site are shown in Fig. 2c. An additional pair of crystal structures of a hammerhead ribozyme sequence found embedded in the original satellite RNA of tobacco ringspot virus (STRSV) has more recently been solved (2QUS,2QUW) [33]. These structures reveal pre-catalytic and post-catalytic complexes of a distinctly different class of hammerhead ribozymes.

\subsection{Two classes of hammerhead ribozymes}

Two classes of tertiary contacts exist in natural hammerhead ribozymes (Fig. 3). These two types of tertiary contacts appear to share very few sequence similarities. One of these classes corresponds to the sTRSV hammerhead, and the other corresponds to the $\operatorname{Sm} \alpha$ hammerhead. Apart from differences in the tertiary contact region itself, a necessary consequence of the very dissimilar sequences, the RNA backbone folds and the three-dimensional structures of the catalytic core regions of the two classes of hammerhead ribozymes are remarkably similar. The sTRSV hammerhead and the Sm $\alpha$ hammerhead tertiary contacts induce what are essentially identical conformational changes in the ribozyme's catalytic core, despite the fact that the sequences and structures of the two tertiary contact regions are radically different from one another.

In both classes of hammerhead tertiary contacts, an apparently conserved Hoogsteen base pair forms between an A in Stem-Loop II and $\mathrm{a} U$ in the non-helical region of Stem I. The A in the Hoogsteen pair corresponds to position 46 in the sTRSV hammerhead and L6 in the Sm $\alpha$ hammerhead, and the U corresponds to position 19 in the STRSV hammerhead and B5 in the Sm $\alpha$ hammerhead. Of the 13 natural hammerhead sequences considered in previous pre-structure modeling studies [36], all possess this final A in the GNRA tetraloop capping Stem II, and 10 possess this $U$ adjacent to residue 1.6, suggesting the AU Hoogsteen pair is conserved due to its functional relevance, despite the fact that it evaded identification before now (the remaining three sequences have $\mathrm{C}$ instead of $\mathrm{U}$, which can form an analogous Hoogsteen pair if the $C$ is protonated).

\section{Riboymes and gene regulation}

\subsection{Ribozymes embedded in mRNAs}

It is now well-known that structured RNAs embedded in untranslated regions (UTRs) of mRNAs can regulate gene expression. Riboswitches have fairly recently been identified as a new class of 

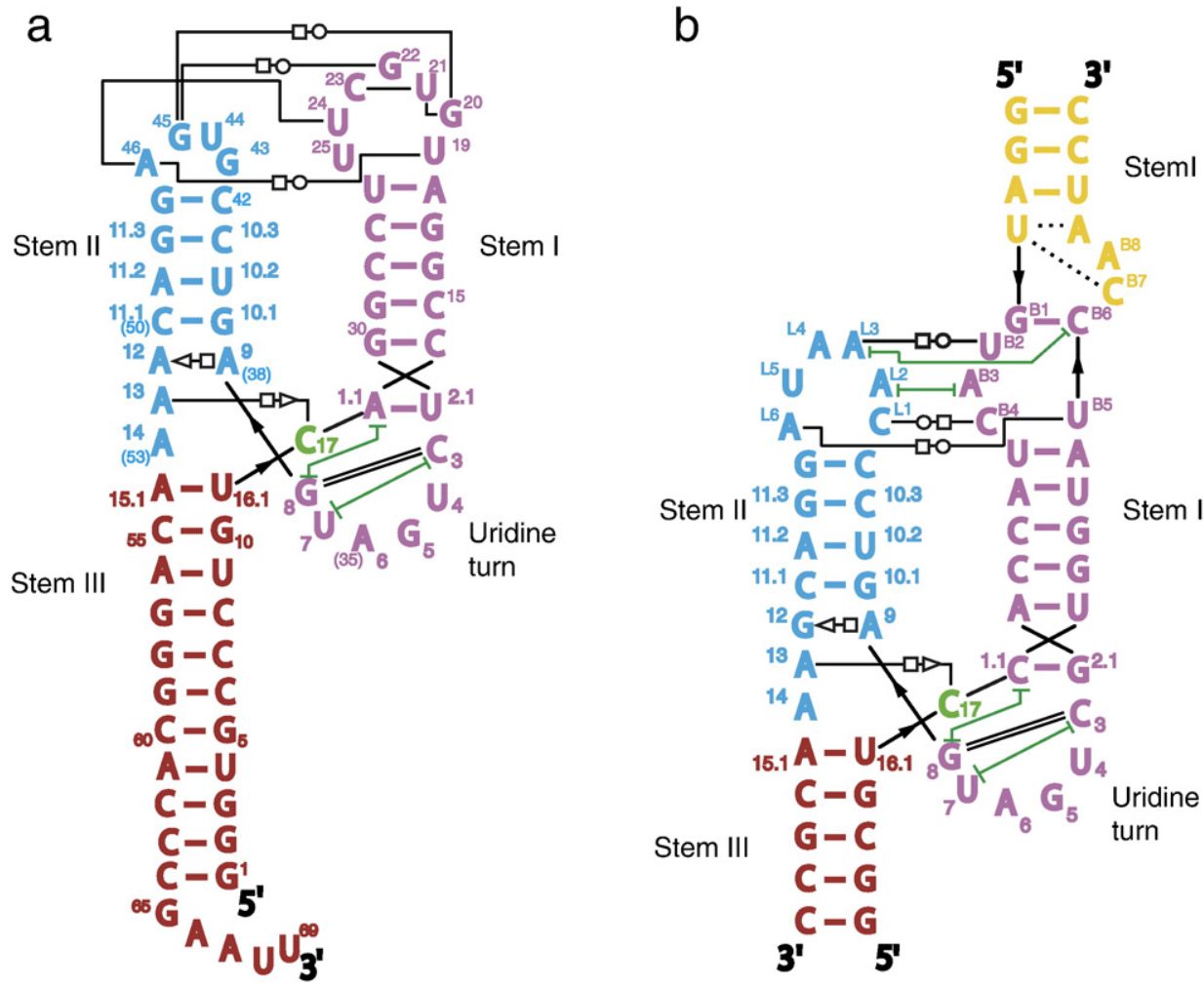

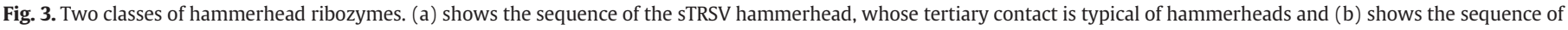
the $\operatorname{Sm} \alpha$ hammerhead.

bacterial gene regulatory elements [13], and the list of these metabolite-binding motifs that typically reside within the $5^{\prime}$ untranslated regions (UTRs) continues to expand. One of these, the glmS riboswitch, is a ribozyme [14], as described in the introductory comments. Upon binding of the metabolite GlcN6P, gene expression is terminated concomitantly with cleavage of the mRNA. As noted in the description of ribozyme acid/base catalysis, GlcN6P is a small molecule ribozyme cofactor that plays the role of the general acid in the mRNA cleavage reaction $[24,25]$.

Riboswitches are thought to be limited to prokaryotic organisms, and the glmS ribozyme was the only example of a known ribozyme that resides embedded within a mature, functional mRNA [37,38]. Group II introns obviously play important regulatory roles in the splicing reactions of several eukaryotic pre-mRNAs [39], but as ribozymes they do not appear to regulate the translational expression of the mature, processed mRNAs from which they have been excised in any obvious way.

It was therefore a rather unexpected discovery to find that hammerhead ribozymes, in some cases, are involved in regulating gene expression in mammals by inducing cleavage within the 3 '-UTR region of the mRNA. Highly active hammerhead ribozymes were discovered embedded within the 3' UTRs of mammalian C-type lectin type II (CLEC2) genes [15]. Hammerhead ribozyme sequences whose invariant regions occur as two fragments (schematically indicated in yellow for substrate and blue for enzyme sequences in Fig. 4a and b) were observed to be separated by hundreds of nucleotides. A fragment pair assembles to form an active hammerhead ribozyme structure between the translation termination and polyadenylation signals within the $3^{\prime}$ UTR. The conserved nucleotides in these hammerhead sequences (shown in red in Fig. 4c) map into the invariant regions of the full-length hammerhead structure. The embedded hammerhead RNA has been shown to self-cleave both in vitro and in vivo, and it is able to reduce protein expression in mouse cells. These results permit us to suggest that a previously unrecognized mechanism of post-transcriptional gene regulation involving association of discontinuous ribozyme sequences within an mRNA may be regulating the expression of several CLEC2 proteins that function in bone remodeling and the immune response of several mammals dating back to platypus. This may constitute but one specific example of a far more general regulatory phenomenon.

\subsection{A hammerhead ribozyme eukaryotic riboswitch?}

Cleavage within the $3^{\prime}$-UTR will remove the polyA tail from a mRNA and will thus interfere with nuclear export as well as the circularization of the message that is believed to be critical to efficient, processive translation. However, if the embedded hammerhead ribozyme always cleaves, the clec2 genes can never be expressed. Hence it seems likely that there exists one or more mechanisms by which the embedded hammerhead is activated or deactivated, in analogy to the nuclease/ligase switch that a genomic hammerhead ribozyme must undergo. In addition, the specific effector for the putative hammerhead riboswitch remains to be identified.

\section{Regulation of catalysis}

4.1. How does the hammerhead ribozyme switch from a nuclease into a ligase?

For the genomic hammerhead self-cleavage motif to function in rolling-circle replication, it must be capable of both cleavage and ligation. A switching mechanism must therefore exist that transforms the hammerhead from a nuclease into a ligase. A nuclease-ligase switch may also be involved in controlling gene expression in the case of the 3'-UTR hammerhead. Therefore, by understanding the structural bases of the nuclease/ligase switch, we may gain insight into how the mRNA-embedded hammerhead regulates gene expression. 
a

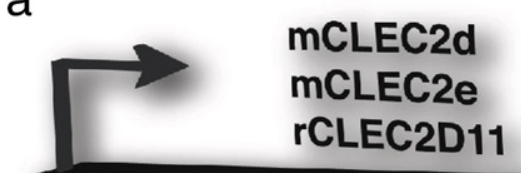

\section{MCLEC20 \\ rCLEC2D11}

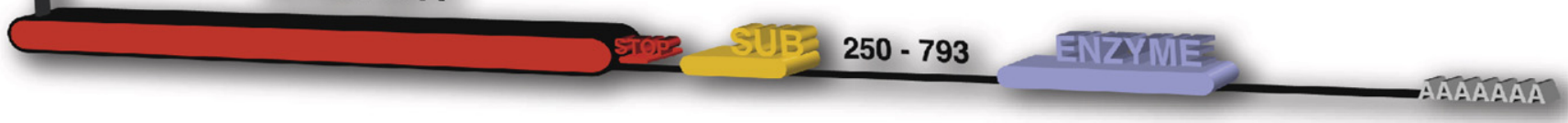

b

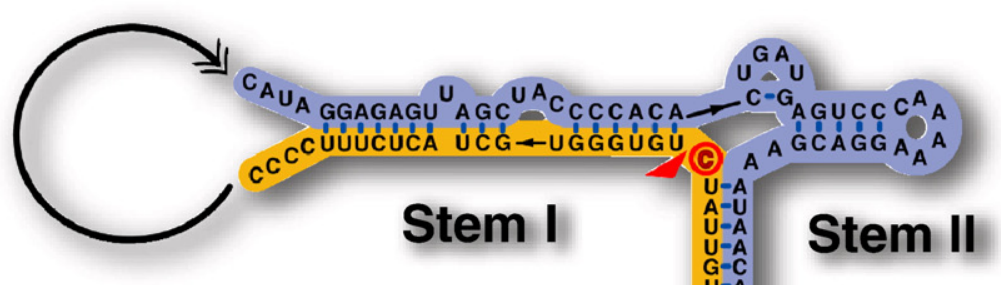

Stem III

CLEC2d

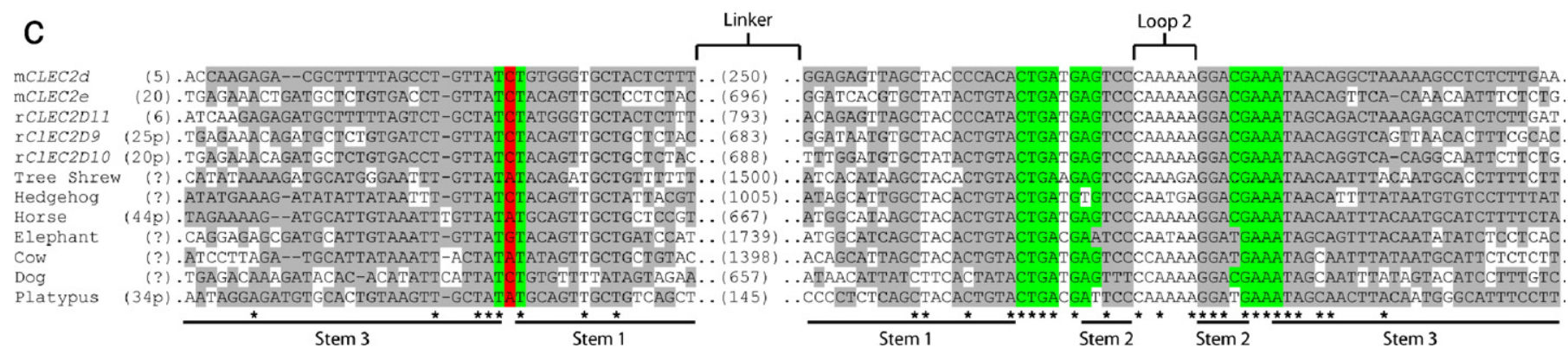

Fig. 4. A discontinuous hammerhead ribozyme embedded in a mammalian mRNA. The substrate (yellow) and enzyme (blue) strands are embedded in the 3'-UTR of the CLEC2d message, separated by a nonconserved 250-793 nt insert. Invariant residues that are critical for catalysis map onto critical regions of the full-length hammerhead structure. The sequence alignment at the bottom reveals similar 3'-UTR hammerheads in a variety of CLEC2-like mammalian mRNAs including platypus.

\subsection{The internal equilibrium of the hammerhead ribozyme}

The hammerhead ribozyme internal equilibrium is defined [40] as the ratio of the concentrations of enzyme-product complex [EP] to enzyme-substrate complex [ES], i.e.,

$K_{\text {int }}=[\mathrm{EP}] /[\mathrm{ES}]$

An efficient nuclease will have a very large $K_{\text {int }}$, whereas an efficient ligase will have a $K_{\text {int }}$ approaching zero.

The first hints that the relative disposition of Stems I and II might effect the internal equilibrium of the hammerhead ribozyme appeared from crosslinking experiments involving the minimal hammerhead sequence that lacked the then unappreciated tertiary contact region $[41,42]$. Chemical crosslinking experiments in the minimal hammerhead ribozyme between Stems I and II, which were designed originally to test between various proposed structures of the minimal hammerhead, yielded an unexpected result. Tethering and constraining the relative motion of these two helices can shift the internal equilibrium of the hammerhead ribozyme away from cleavage and toward ligation. A series of crystal structures of a tethered minimal hammerhead also revealed a possible structural basis for the shift in equilibrium. Compared to unrestrained hammerhead crystal structures, the tethered hammerhead that favored ligation revealed a change in helical twist in Stem I relative to the remainder of the ribozyme molecule. Untethered minimal hammerheads typically have an internal equilibrium constant that greatly favors cleavage product formation.

Full-length hammerhead ribozymes have a tertiary contact between Stem loops I and II that, in some cases, appears to impart an effect somewhat similar to tethering and constraining these helices in the minimal hammerhead. Although the internal equilibrium in a hammerhead ribozyme derived from the satellite RNA of tobacco ringspot virus (sTRSV) appears to strongly favor product formation [43], the internal equilibrium of the Schistosomal ( $\operatorname{Sm\alpha }$ ) hammerhead has a $K_{\text {int }} \sim 3 / 1$; i.e., it has significant ligase activity [44]. 
A comparison between the $\operatorname{Sm} \alpha$ hammerhead structure and the sTRSV hammerhead structures reveals a change in Stem I helical twist in otherwise very similar tertiary structures. This change is rather similar to what had been observed in the case of the tethered hammerhead, permitting us to hypothesize that differences in the two types of tertiary contacts in these full-length hammerheads will give rise to different internal equilibrium constants via conformational changes propagated through the Stem I helix. Fig. 5 depicts this comparison (the $\operatorname{Sm} \alpha$ hammerhead, which favors ligation more than the others, is shown in magenta). Despite two very different crystal packing environments of the two crystallographically independent molecules in the sTRSV ribozyme crystals, the conformations of both sTRSV hammerhead Stem I helices are nearly identical, whereas the difference between the sTRSV and the $\operatorname{Sm} \alpha$ hammerhead is much more pronounced. This observation permits us to suggest the differences cannot be explained away as crystal packing artifacts; the two crystallographically independent sTRSV hammerheads serve as a positive control.

\subsection{A proposed switching mechanism}

Comparison of the limited sequence and structural conservation within the tertiary contact region of both full-length hammerhead classes reveals how two conformational switches activate the hammerhead ribozyme for catalysis and regulate the nuclease-ligase internal equilibrium that is critical within the rolling circle replicative cycle of RNA viroids and satellite virus RNAs.

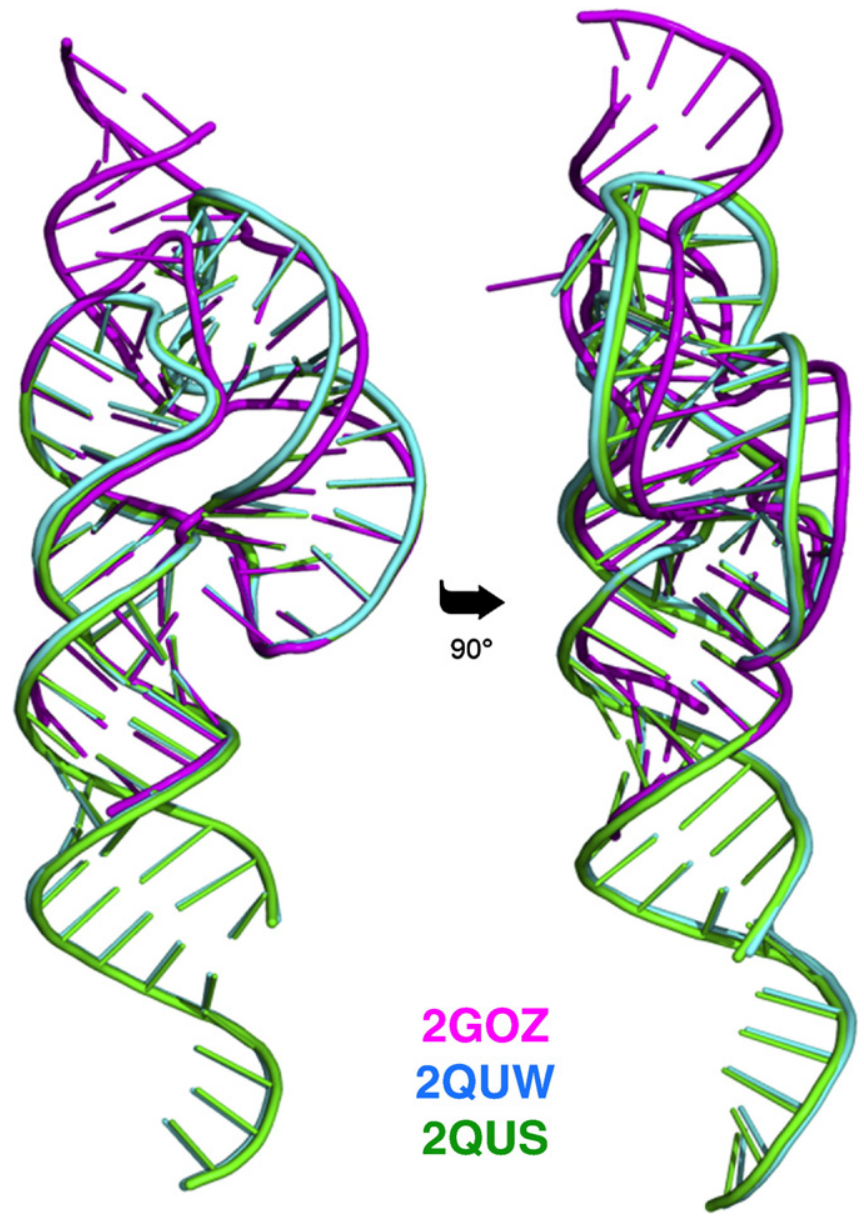

Fig. 5. A comparison between our the Sm $\alpha 1$ hammerhead structure and the sTRSV hammerhead structure reveals a change in Stem I helical twist in otherwise very similar tertiary structures. The $S m \alpha 1$ hammerhead, which favors ligation more than the others, is shown in magenta. The shift of $K_{\text {int }}$ toward ligation appears to correspond to anticlockwise twisting of Stem I relative to the rest of the ribozyme.
The crystallographic comparison of the two hammerhead classes, together with these internal equilibrium experiments, permit us to suggest that the detailed structure of the tertiary contact region modulates the internal equilibrium of the hammerhead ribozyme, thus functioning as one of at least two molecular switches that regulates hammerhead ribozyme catalytic activity. A second switch, which serves to activate the ribozyme, includes the previously described formation of a Watson-Crick tertiary base pair between the invariant C3 and G8 residues in the hammerhead's catalytic core.

4.3.1. Conformational switching within the hammerhead tertiary contact Although the involvement of conserved GNRA tetraloops in docking interactions is frequently observed in a variety of RNA structures [45-47], it is particularly noteworthy that the tertiary contact observed in the sTRSV hammerhead structure can only form if the tetraloop rearranges from its canonical and energetically favored conformation (Fig. 6a) to expose the Hoogsteen face of the final A to its hydrogen-bonding partner in Stem I (Fig. 6b). This immediately suggests that a thermodynamic competition must exist between an isolated GNRA tetraloop capping Stem II that adopts the canonical conformation (Fig. 6a), as seen in the original hammerhead structure $[46,48]$, and one that interacts with Stem I, where the conserved U can only form a Hoogsteen pair when the GNRA tetraloop adopts the observed non-canonical conformation (Fig. 6b). This observation contrasts with tetraloop-receptor interactions observed in other RNA structures, such as RNase P, the Group I intron and the ribosome, wherein the tetraloop typically retains the canonical conformation. In the case of natural hammerhead RNAs, the interface formed between the tetraloop and its target sequence on Stem I may be rather more dynamic. In addition, the target sequence on Stem I may also be dynamic. In many of the natural sequences, the conserved U in Stem I that forms a Hoogsteen pair with the A in Stem II also has the option of forming a canonical AU or wobble GU pair that would simply extend Stem I at the expense of forming the tertiary contact. Hence both the tetraloop and its target sequence likely exist in multiple conformational states. Tetraloop conformational dynamics have in fact been implicated previously in the cleavage-ligation switching mechanism of potato spindle tuber viroid, a species whose cleavage and ligation reactions are protein-dependent [49].

\subsubsection{Conformational switching in the hammerhead catalytic core}

A second conformational switch takes place, within the hammerhead ribozyme's active site, whose arrangement in the full-length hammerhead differs radically from that of the minimal hammerhead. In the absence of the stabilizing tertiary contact between Stems I and II, the minimal hammerhead crystal structure likely represents the dominant species, and transient, low-occupancy fluctuations rearrange the active site for catalysis. This rearrangement, stabilized by the tertiary contact in the full-length ribozyme, appears as a consequence of the invariant G8 rotating from a position (Fig. 6c) in which it forms a sheared base-pair with A13 of Stem II in the minimal hammerhead structure to a position in which it forms a Watson-Crick base-pair with the invariant C3 of Stem I (Fig. 6d) in the catalytically competent full-length structure. This conformational change is in turn responsible for positioning the cleavage-site nucleotide for in-line attack, and positions G12 to function as the general base in catalysis.

\subsubsection{Conformational switching, internal equilibria, and catalysis}

Conformational switches in both the tertiary contact region and the active site thus appear to act cooperatively to stabilize the hammerhead ribozyme in the active conformation. Loss of the tertiary contact switch results in a ribozyme that is approximately 1000 -fold less catalytically active.

Although the tertiary interaction between Stems I and II in the fulllength hammerhead greatly accelerates both the cleavage and ligation reaction rates, the ligation rate is accelerated much more. The internal 
a
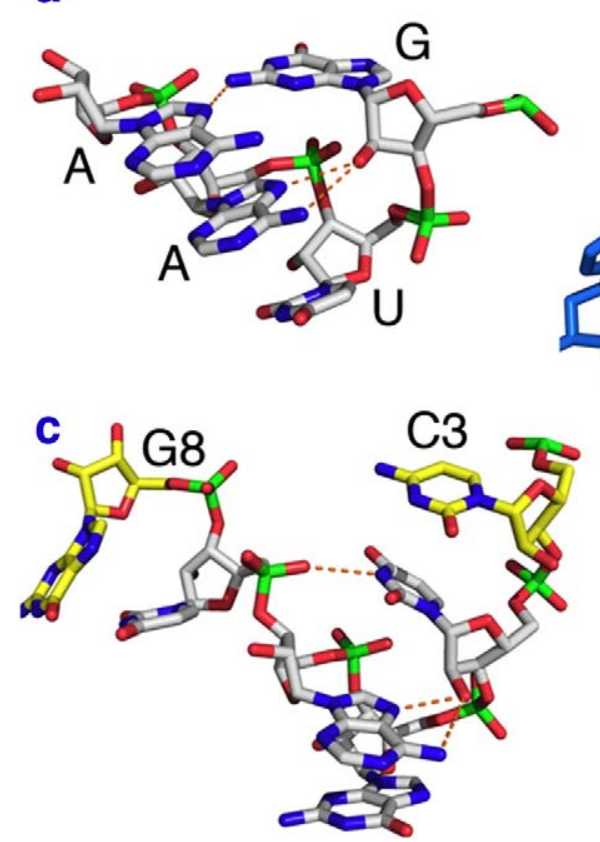

b
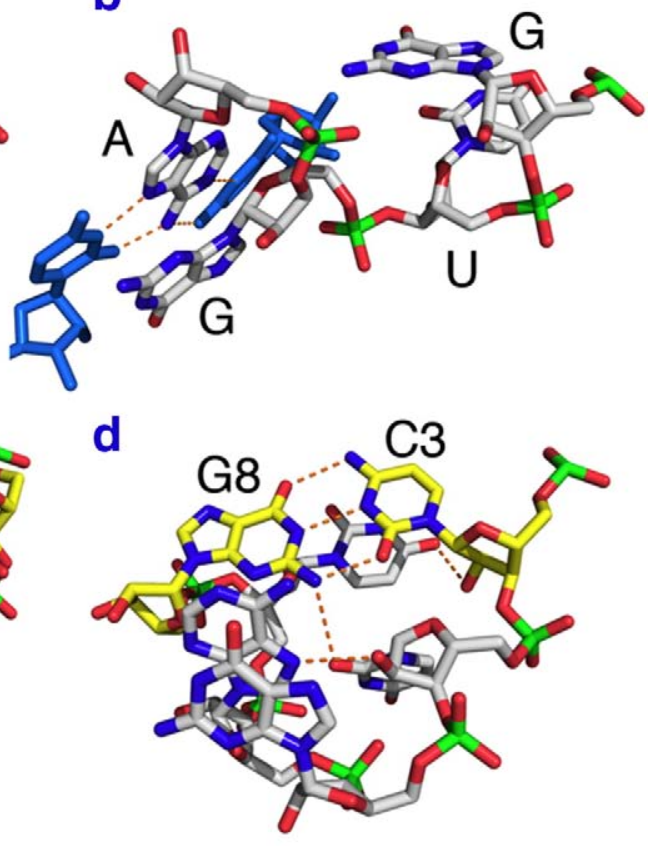

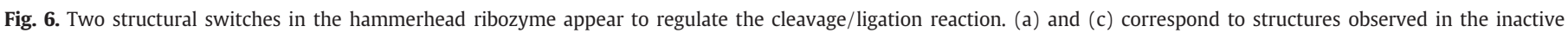

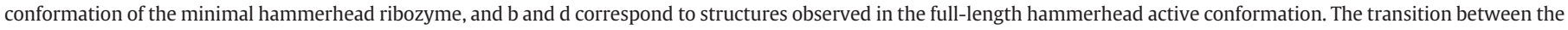

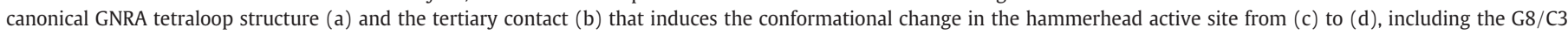
pairing interaction.

equilibrium of the $S m \alpha$ hammerhead is such that $1 / 3$ of the substrate is ligated, whereas most is cleaved in constructs derived from STRSV hammerhead. At least two sets of observed differences between the two classes of hammerheads suggest what may be responsible. Since formation of the tertiary contact modulates the internal equilibrium of the hammerhead reaction, a simple nuclease-ligase switching mechanism would result as a consequence of the observed dual conformational states of the tetraloop on Stem II and its complementary target structure in Stem I in the sTRSV RNA, whereas there are no obvious alternate conformations corresponding to the tertiary contact region within the $S m \alpha$ ribozyme. In addition, the internal equilibrium between cleavage and ligation of bound substrate may also be finetuned by the orientation of Stem I relative to Stem II. Previously, it has been observed that crosslinking Stems I and II in a minimal hammerhead construct can differentially alter the internal equilibrium of the hammerhead as a function of relative helix orientation. Superposition of the sequences shared between the $\operatorname{Sm} \alpha$ hammerhead and the STRSV hammerhead (Fig. 5) in fact reveals the STRSV hammerhead Stem I to be significantly more tightly wound than that of the Sm $\alpha$ hammerhead, with the sTRSV substrate strand more tightly kinked at the cleavage site. Hence it is likely that the observed differences between the sTRSV and $\operatorname{Sm} \alpha$ hammerhead Stem I orientations correlate with the observed differences in internal equilibria. Noteably, the cleaved and uncleaved forms of the sTRSV are far more similar to each other (Fig. 5) than either is to the Sm $\alpha$ hammerhead, a hammerhead that, as noted, tends to favor ligation more than the STRSV. The structural details of two molecular switch sites now have been identified in the hammerhead ribozyme structure. The tertiary contact between Stems I and II, which permits formation of a newly identified conserved AU Hoogsteen base pair, activates the ribozyme approximately 1000 -fold when it is formed by the interaction of two dynamic loops that can exist in multiple conformational states. This switch in turn induces formation of a second, previously identified, tertiary contact between G8 and C3 in the catalytic core that locks the highly dynamic active site structure into a catalytically competent conformation. Together, these switches can activate or deactivate hammerhead ribozyme catalysis. Activation of catalysis in this way has a concomitant effect of accelerating the ligation reaction rate to a greater extent than the cleavage rate.

However, the Stem I-Stem II tertiary contact switch (Fig. 6a and b) is not simply binary (i.e., it is not simply based on a dissociation equilibrium), but rather it appears also to further modulate the internal equilibrium of the hammerhead ribozyme as a molecular rheostat. Specifically, the tertiary contact region that surrounds the AU Hoogsteen base pair between the enzyme and substrate differs in the two classes of hammerhead RNAs, and this contextual variance results in a changeable helical pitch of Stem I between the cleavage site and the tertiary contact (Fig. 5). Stem I in the sTRSV hammerhead is more tightly wound than it is in the Sm $\alpha$ hammerhead, suggesting that Stem I helical unwinding shifts the hammerhead internal equilibrium toward ligation. Whether Stem I helical winding and unwinding can occur within a specific hammerhead sequence is still unclear, but the propensity for internal equilibrium adjustment via changes within the structural context of the tertiary contact region is clear.

\section{References}

[1] K. Kruger, P.J. Grabowski, A.J. Zaug, J. Sands, D.E. Gottschling, T.R. Cech, Selfsplicing RNA: autoexcision and autocyclization of the ribosomal RNA intervening sequence of Tetrahymena, Cell 31 (1982) 147-157.

[2] C. Guerrier-Takada, K. Gardiner, T. Marsh, N. Pace, S. Altman, The RNA moiety of ribonuclease P is the catalytic subunit of the enzyme, Cell 35 (1983) 849-857.

[3] G.A. Prody, J.T. Bakos, J.M. Buzayan, I.R. Schneider, G. Breuning, Autolytic processing of dimeric plant virus satellite RNA, Science 231 (1986) 1577-1580.

[4] A. Hampel, R. Tritz, RNA catalytic properties of the minimum (-)sTRSV sequence, Biochemistry 28 (1989) 4929-4933.

[5] M.Y. Kuo, L. Sharmeen, G. Dinter-Gottlieb, J. Taylor, Characterization of selfcleaving RNA sequences on the genome and antigenome of human hepatitis delta virus, J. Virol. 62 (1988) 4439-4444.

[6] T.A. Steitz, P.B. Moore, RNA, the first macromolecular catalyst: the ribosome is a ribozyme, Trends Biochem. Sci. 28 (2003) 411-418.

[7] N. Ban, P. Nissen, J. Hansen, P.B. Moore, T.A. Steitz, The complete atomic structure of the large ribosomal subunit at 2.4 A resolution, Science 289 (2000) 905-920.

[8] P. Nissen, J. Hansen, N. Ban, P.B. Moore, T.A. Steitz, The structural basis of ribosome activity in peptide bond synthesis, Science 289 (2000) 920-930.

[9] M.M. Yusupov, G.Z. Yusupova, A. Baucom, K. Lieberman, T.N. Earnest, J.H. Cate, H.F. 
Noller, Crystal structure of the ribosome at 5.5 A resolution, Science 292 (2001) 883-896.

[10] J.H. Cate, M.M. Yusupov, G.Z. Yusupova, T.N. Earnest, H.F. Noller, X-ray crystal structures of 70S ribosome functional complexes, Science 285 (1999) 2095-2104.

[11] M. Selmer, C.M. Dunham, F.V.T. Murphy, A. Weixlbaumer, S. Petry, A.C. Kelley, J.R Weir, V. Ramakrishnan, Structure of the 70S ribosome complexed with mRNA and tRNA, Science 313 (2006) 1935-1942.

[12] H.F. Noller, V. Hoffarth, L. Zimniak, Unusual resistance of peptidyl transferase to protein extraction procedures, Science 256 (1992) 1416-1419.

[13] W. Winkler, A. Nahvi, R.R. Breaker, Thiamine derivatives bind messenger RNAs directly to regulate bacterial gene expression, Nature 419 (2002) 952-956.

[14] W.C. Winkler, A. Nahvi, A. Roth, J.A. Collins, R.R. Breaker, Control of gene expression by a natural metabolite-responsive ribozyme, Nature 428 (2004) 281-286.

[15] M. Martick, L.H. Horan, H.F. Noller, W.G. Scott, A discontinuous hammerhead ribozyme embedded in a mammalian messenger RNA, Nature 454 (2008) 899-902.

[16] W.G. Scott, Ribozymes, Curr. Opin. Struct. Biol. 17 (2007) 280-286.

[17] J.C. Cochrane, S.A. Strobel, Catalytic strategies of self-cleaving ribozymes, Acc. Chem. Res. 41 (2008) 1027-1035.

[18] J.B. Murray, A.A. Seyhan, N.G. Walter, J.M. Burke, W.G. Scott, The hammerhead, hairpin and VS ribozymes are catalytically proficient in monovalent cations alone, Chem. Biol. 5 (1998) 587-595.

[19] R.T. Raines, RibonucleaseA , Chem. Rev. 98 (1998) 1045-1066.

[20] A. Wlodawer, M. Miller, L. Sjölin, Active site of RNase: neutron diffraction study of a complex with uridine vanadate, a transition-state analog, Proc. Natl. Acad. Sci. U. S. A. 80 (1983) 3628-3631.

[21] M.J. Fedor, Comparative enzymology and structural biology of RNA self-cleavage, Annu. Rev. Biophys. 38 (2009) 271-299.

[22] P.B. Rupert, A.P. Massey, S.T. Sigurdsson, A.R. Ferré-D'Amaré, Transition state stabilization by a catalytic RNA, Science 298 (2002) 1421-1424.

[23] M. Martick, W.G. Scott, Tertiary contacts distant from the active site prime a ribozyme for catalysis, Cell 126 (2006) 309-320.

[24] J.C. Cochrane, S.V. Lipchock, S.A. Strobel, Structural Investigation of the GlmS Ribozyme Bound to Its Catalytic Cofactor, Chem. Biol. (2006).

[25] D.J. Klein, A.R. Ferre-D'Amare, Structural basis of glmS ribozyme activation by glucosamine-6-phosphate, Science 313 (2006) 1752-1756.

[26] R.H. Symons, Plant pathogenic RNAs and RNA catalysis, Nucleic Acids Res. 25 (1997) 2683-2689.

[27] R. Flores, M.E. Gas, D. Molina, C. Hernández, J.A. Darós, Analysis of viroid replication, Methods Mol. Biol. 451 (2008) 167-183.

[28] G. Ferbeyre, J.M. Smith, R. Cedergren, Schistosome satellite DNA encodes active hammerhead ribozymes, Mol. Cell. Biol. 18 (1998) 3880-3888.

[29] G. Ferbeyre, V. Bourdeau, M. Pageau, P. Miramontes, R. Cedergren, Distribution of hammerhead and hammerhead-like RNA motifs through the GenBank, Genome Res. 10 (2000) 1011-1019.

[30] P.B. Rupert, A.R. Ferré-D'Amaré, Crystal structure of a hairpin ribozyme-inhibitor complex with implications for catalysis, Nature 410 (2001) 780-786.
[31] A.R. Ferré-D'Amaré, K. Zhou, J.A. Doudna, Crystal structure of a hepatitis delta virus ribozyme, Nature 395 (1998) 567-574.

[32] A. Ke, K. Zhou, F. Ding, J.H. Cate, J.A. Doudna, A conformational switch controls hepatitis delta virus ribozyme catalysis, Nature 429 (2004) 201-205.

[33] Y.I. Chi, M. Martick, M. Lares, R. Kim, W.G. Scott, S.H. Kim, Capturing hammerhead ribozyme structures in action by modulating general base catalysis, PLoS Biol. 6 (2008) e234.

[34] W.G. Scott, Biophysical and biochemical investigations of RNA catalysis in the hammerhead ribozyme, Q. Rev. Biophys. 32 (1999) 241-284.

[35] M. De la Peña, S. Gago, R. Flores, Peripheral regions of natural hammerhead ribozymes greatly increase their self-cleavage activity, EMBO J. 22 (2003) 5561-5570.

[36] A. Khvorova, A. Lescoute, E. Westhof, S.D. Jayasena, Sequence elements outside the hammerhead ribozyme catalytic core enable intracellular activity, Nat. Struct. Biol. 10 (2003) 708-712.

[37] T.E. Edwards, D.J. Klein, A.R. Ferré-D'Amaré, Riboswitches: small-molecule recognition by gene regulatory RNAs, Curr. Opin. Struct. Biol. 17 (2007) 273-279.

[38] C. Hammann, E. Westhof, Searching genomes for ribozymes and riboswitches, Genome Biol. 8 (2007) 210

[39] O. Fedorova, L.J. Su, A.M. Pyle, Group II introns: highly specific endonucleases with modular structures and diverse catalytic functions, Methods 28 (2002) 323-335.

[40] K.J. Hertel, O.C. Uhlenbeck, The internal equilibrium of the hammerhead ribozyme reaction, Biochemistry 34 (1995) 1744-1749.

[41] S.T. Sigurdsson, T. Tuschl, F. Eckstein, Probing RNA tertiary structure: interhelical crosslinking of the hammerhead ribozyme, Rna 1 (1995) 575-583.

[42] K.F. Blount, O.C. Uhlenbeck, Internal equilibrium of the hammerhead ribozyme is altered by the length of certain covalent cross-links, Biochemistry 41 (2002) 6834-6841.

[43] J.A. Nelson, I. Shepotinovskaya, O.C. Uhlenbeck, Hammerheads derived from sTRSV show enhanced cleavage and ligation rate constants, Biochemistry 44 (2005) 14577-14585.

[44] M.D. Canny, F.M. Jucker, A. Pardi, Efficient ligation of the Schistosoma hammerhead ribozyme, Biochemistry 46 (2007) 3826-3834.

[45] L. Jaeger, F. Michel, E. Westhof, Involvement of a GNRA tetraloop in long-range RNA tertiary interactions, J. Mol. Biol. 236 (1994) 1271-1276.

[46] H.W. Pley, K.M. Flaherty, D.B. McKay, Model for an RNA tertiary interaction from the structure of an intermolecular complex between a GAAA tetraloop and an RNA helix, Nature 372 (1994) 111-113.

[47] D.L. Abramovitz, A.M. Pyle, Remarkable morphological variability of a common RNA folding motif: the GNRA tetraloop-receptor interaction, J. Mol. Biol. 266 (1997) 493-506.

[48] H.W. Pley, K.M. Flaherty, D.B. McKay, Three-dimensional structure of a hammerhead ribozyme, Nature 372 (1994) 68-74.

[49] T. Baumstark, A.R. Schröder, D. Riesner, Viroid processing: switch from cleavage to ligation is driven by a change from a tetraloop to a loop E conformation, EMBO J. 16 (1997) 599-610. 